\title{
Après la catastrophe, bifurquer ou persévérer? Les forestiers à l'épreuve des événements climatiques extrêmes
}

\author{
Vincent Banos ${ }^{1, *}$ et Philippe Deuffic ${ }^{2}$ \\ 1 Géographie, INRAE, UR ETBX, Cestas, France \\ ${ }^{2}$ Sociologie, INRAE, UR ETBX, Cestas, France
}

Reçu le 18 juillet 2018. Accepté le 2 janvier 2020

L'arrivée de la catastrophe nous obligera à changer radicalement nos visions et nos pratiques, entend-on souvent dans la bouche de ceux qui dénoncent le caractère insoutenable de nos dynamiques économiques et sociales. Mais en est-on bien sûr? Les choses n'apparaissent, en effet, pas si simple, comme le montrent les auteurs. Leur analyse rétrospective des modes de gestion forestiers dans les Pyrénées audoises et les Landes de Gascogne conclut que la sécheresse de 2003 et la tempête Klaus de 2009 qui ont frappé ces deux massifs n'ont pas conduit à leur remise en cause, mais plutôt à une accélération des évolutions des stratégies et des pratiques des propriétaires qui étaient déjà en cours. C'est le cas notamment, dans la forêt landaise, de la mobilisation de la ressource en bois-énergie constituée par les souches, comme l'avaient montré V. Banos et J. Dehez dans un précédent article publié dans $\operatorname{NSS}(2017,25,2)$.

La Rédaction

Résumé - En dépit de l'attention croissante accordée aux catastrophes climatiques, leur influence réelle sur les changements de pratiques reste ambivalente. Pour contribuer à ce débat, nous questionnons les stratégies d'adaptation adoptées par des propriétaires forestiers suite à la sécheresse de 2003 (Aude) et la tempête de 2009 (Landes de Gascogne). L'hypothèse défendue est que ces catastrophes n'ont conduit ni à la révolution, ni au statu quo mais à une « bifurcation assistée ». En effet, les stratégies mises en œuvre dix ans après oscillent entre retour aux routines et intensification des pratiques sylvicoles. Ces changements dans la continuité s'expliquent notamment par le fait que l'autorité des experts, loin d'être ébranlée, fut renforcée par les processus de sortie de crise. Au final, cet article souligne l'intérêt de penser les catastrophes, non pas seulement comme des risques à venir, mais comme des laboratoires permettant d'éprouver la pertinence et l'efficacité des réflexions sur l'adaptation.

Mots-clés : environnement / risques / disaster studies / changement de pratiques / forêt

\begin{abstract}
After the disaster: change direction or carry on? French forest owners cope with extreme climatic events. Although climatic disasters have been scrutinized for years, their impacts on changes in practices are unclear. Our contribution aims to question the adaptive strategies implemented by private forest owners following two major natural disasters: storm Klaus in 2009 (Landes de Gascogne Forest) and a severe drought in 2003 (Pays de Sault Forest). We hypothesize that the outcome of these disasters was neither a revolution nor a status quo but rather a so-called 'assisted rerouting'. Our analysis shows that, ten years after these events, strategies have ranged from a return to routine to intensification of sylvicultural practices. The continuity observed despite these changes is due in particular to the experts' epistemic authority, which, far from being challenged, was strengthened by the crisis-solving process. Finally, we suggest that such disasters should not be described only from the angle of future risks, but considered also as experiments that test the relevance and efficiency of adaptive strategies.
\end{abstract}

Keywords: environment / risk / disaster studies / changes in practices / forest

*Auteur correspondant : vincent.banos@inrae.fr 
De Katrina à Fukushima en passant par Xynthia, les catastrophes opèrent une rupture franche de l'ordre social et économique (Chateauraynaud et Torny, 2013). Là où, d'ordinaire, le rapport entre les mots et les choses coulait de source, elles créent un grippage et un décadrage des récits interprétatifs classiques (Moreau, 2017). La recherche d'un sens à donner à la catastrophe et d'un enseignement à en tirer milite alors activement pour que l'événement ait un avant et un après, un «plus jamais ça » ou « un plus rien ne sera comme avant» (Journet, 2010). Ces événements extrêmes constitueraient des fenêtres d'opportunité pour instiller des changements radicaux de politiques et de pratiques (Kingdon, 1995). Pourtant, la portée de ces changements et l'influence réelle des catastrophes demeurent bien incertaines (Henry, 2011). Cette difficulté à apprécier le rôle exact des crises fait écho à des débats en sociologie sur le poids respectif des événements conjoncturels et celui des tendances structurelles à long terme dans les conduites humaines (Bessin et al., 2010). Les observateurs spécialisés émettent ainsi l'idée que les chocs produisent du changement mais aussi de la stabilité, du conflit et en même temps du consensus (Quarantelli et Dynes, 1977). Pour d'autres, les catastrophes perpétuent un ordre du monde mais avec des aménagements possibles (Clavandier, 2015), précision sibylline qui illustre la persistance d'une ambivalence.

Cette ambiguïté se retrouve aussi dans le monde forestier. Dans ce milieu souvent marqué par le poids des routines et des traditions familiales (MAAF, 2014), les tempêtes de 1999 et 2009 ou la sécheresse de 2003 ont été perçues comme des catastrophes susceptibles d'entraîner de profonds changements. Ces événements climatiques extrêmes (ECE) ont en effet détruit plusieurs milliers d'hectares de forêt, ils ont frappé indistinctement petits et grands propriétaires et ont profondément ébranlé leurs convictions. Ils ont donné du poids à l'idée qu'il était nécessaire de préparer les forêts au changement climatique (Roman-Amat, 2007). Pourtant, dix ou quinze ans plus tard, les propriétaires ont certes fait évoluer leurs pratiques mais pas autant que ce qui avait été envisagé au lendemain de ces catastrophes. Pourquoi et comment la révolution qui s'annonçait s'est-elle transformée en une «bifurcation assistée »? Comment et par qui les quelques changements initiés ont-ils été orientés et au nom de quoi? Pour répondre à ces questions, nous nous appuyons sur l'analyse de deux ECE: la tempête Klaus de 2009, qui a ravagé la forêt landaise, et la sécheresse de 2003, qui a provoqué des dépérissements massifs dans les Pyrénées audoises (Fig. 1 et 2). Nous verrons comment ces catastrophes ont ouvert un champ de questionnements relativement large et inédit puis comment celui-ci s'est refermé. Nous identifierons également les facteurs qui ont contribué à un changement dans la continuité plutôt qu'à un bouleversement radical des pratiques de gestion forestière.

\section{Problématique et cadre d'analyse}

L'effet des catastrophes sur les pratiques et les comportements routiniers restant controversé, il s'agit de faire le point sur les arguments avancés en sociologie, puis dans le domaine plus spécialisé des disaster studies, avant de voir en quoi les débats au sein de la sphère forestière prolongent ce questionnement.

\section{La catastrophe, une conjoncture qui déstructure?}

Alors que les approches biographiques foisonnent d'exemples où les individus témoignent d'une rupture radicale au cours de leur vie, la sociologie reste un peu désarmée sur le plan théorique pour donner du sens à l'idée qu'une séquence d'actions à l'issue plus ou moins imprévisible puisse avoir des conséquences «importantes » (Grossetti, 2006). Cette discipline s'est en effet longtemps méfiée des événements en considérant que la contingence devait être mise à distance pour analyser les « vraies » causes de nature structurelle (Bensa et Fassin, 2002). Le modèle du cheminement «bifurcatif» rappelle néanmoins que chaque existence se partage entre des moments calmes, où les choses suivent leurs cours, et des moments décisifs où tout est remis en jeu (Coninck et Godard, 1990). Les catastrophes incarnent ces moments de basculement et entrent dans la sous-catégorie relativement rare des chocs qui transforment significativement les structures (Sewell, 1996). Pour être qualifié de «tournant», un changement court et soudain doit cependant déboucher sur une période caractérisée par un nouveau régime d'action (Abbott, 2001). Appliqué à notre objet d'étude, une tempête ou une sécheresse, même exceptionnelle, ne constitue donc pas automatiquement un point de rupture à moins qu'elle induise un véritable changement de paradigme dans la gestion forestière. Toutefois, Grossetti (2006) rappelle que les changements dans les parcours de vie ne se définissent pas uniquement par un contraste absolu entre des phases où rien ne change et d'autres où tout serait possible, mais par le fait que tout peut changer tout le temps à des coûts plus ou moins élevés. Cette approche élargit la notion de bifurcation au-delà des idées d'alternative ou de «tournant» et se rapproche fortement du concept «d'épreuve», très présent dans la sociologie pragmatique (Bessin et al., 2010). Dans les deux cas, il s'agit de désigner un moment d'incertitude débouchant sur des changements de plus ou moins grande ampleur (Grossetti, 2006; Chateauraynaud et Torny, 2013). Le travail de Dobry (1983) sur les crises politiques nourrit également cette perspective puisqu'il montre comment des événements contingents travaillent des structures et des rapports sociaux caractérisés par une certaine plasticité. Tout en reconnaissant que l'issue de ces 
situations de troubles n'est jamais indépendante des places et des ressources sociales des acteurs, ces différentes sociologies promeuvent une conception dynamique et ouverte de la vie sociale. Pour autant, d'autres courants théoriques demeurent plus sceptiques. Tel est le cas du champ des sustainability transitions qui questionne les conditions de transformations sociales et techniques en contexte de crise écologique et climatique (Geels et Schot, 2007; Markard et al., 2012). Tout en admettant que des chocs exogènes peuvent constituer des fenêtres d'opportunités, cette littérature insiste sur la stabilité et le verrouillage des systèmes sociotechniques - par exemple, une filière. Elle considère ainsi que les innovations doivent émerger aux marges des régimes existants et être accompagnées pour résister aux pressions de sélection. Dans cette vision évolutionniste et technocentrée du changement, les événements conjoncturels occupent une place somme toute marginale.

\section{Les disaster studies, la catastrophe au cœur du changement social ?}

Si la sociologie demeure hésitante malgré un intérêt croissant pour les crises et les catastrophes, qu'en est-il de champs d'études plus spécialisés tels que les disasters studies qui placent la question de l'ordre social au cœur de leurs préoccupations originelles (Dodier, 2013)? Pour les précurseurs de ce courant, les catastrophes ouvrent en effet un cycle de destruction-reconstruction favorable à la transformation des communautés impactées (Carr, 1932). Pour autant, les études suivantes montrent que les potentialités de changement dépendent de nombreuses variables telles que l'intensité des événements, la rapidité des secours, la durée de la crise ou encore la capacité de résilience des individus et des communautés touchées (Bates et Peacock, 1987). Accordant une attention particulière aux comportements des victimes, elles soulignent que les réactions rationnelles et les désirs d'un retour aux routines ordinaires l'emportent bien souvent sur la panique, la désagrégation des liens, mais aussi sur l'expérimentation (Dynes et Drabek, 1994). Quarantelli et Dynes (1977) jugent d'ailleurs que les actions menées post-catastrophe prolongent la plupart du temps les logiques préexistantes. La reconnaissance de ce «principe de continuité» est révélatrice d'une reconfiguration des disaster studies autour des enjeux du risque et de la vulnérabilité (Cabane et Revet, 2015). L'intérêt pour les catastrophes comme phénomènes extérieurs aux effets spécifiques s'estompe ainsi au profit d'une déconstruction de leurs «causes profondes» (inégalités socioéconomiques, facteurs politiques...). Cette lecture constructiviste des risques s'accompagne d'un regard critique sur le gouvernement des catastrophes (Cabane, 2012; Revet et Langumier, 2013), du déficit d'information aux victimes à la confiscation des débats par les experts en passant par «le pouvoir du pouvoir» en situation d'urgence (Gilbert, 1992). Pourtant, une certaine ambivalence demeure car, tout en insistant sur l'emprise croissante des dispositifs de contrôle, Revet et Langumier (2013) invitent à davantage questionner ce que les catastrophes contribuent à produire dans les pratiques ordinaires et à faire advenir comme recompositions sociales. Quant à Gilbert (2013), il considère que la multiplication de risques (technologiques, sanitaires et climatiques...) toujours plus porteurs d'incertitudes et d'investissements inattendus redonne un horizon au questionnement sur les crises et leur potentiel de «débordement» de l'ordre social. Au final, rares sont ceux qui rejettent catégoriquement le postulat des pères fondateurs et beaucoup laissent planer le doute quant aux effets des catastrophes catastrophes (Clavandier, 2015; Henry, 2011).

\section{La catastrophe en forêt, frein ou accélérateur de changement?}

Dans le domaine sylvicole, les sciences forestières abordent, dès le $\mathrm{XIX}^{\mathrm{e}}$ siècle, la question des catastrophes à travers la notion de «météores", ce qui justifiera notamment des politiques de reboisement censées en limiter les effets (Decocq et al., 2016). Quant aux incertitudes, elles seront réduites tout au long du $\mathrm{XX}^{\mathrm{e}}$ siècle grâce à l'apport des outils statistiques et prédictifs qui, sauf précisément dans le cas des accidents majeurs, modélisent la croissance des arbres et leur rendement sur plusieurs décennies. Or, en ce début de $\mathrm{XXI}^{\mathrm{e}}$ siècle, le changement climatique met à mal ces modèles biologiques et économiques probabilistes (Lawrence, 2017). La succession de catastrophes en forêt -mégafeux, tempêtes quasi cycloniques, sécheresses à répétition- suscite ainsi de plus en plus d'inquiétude et de perplexité chez les propriétaires forestiers car celles-ci ne relèveraient plus du hasard mais seraient les signes annonciateurs d'un climat en train de changer (Deuffic et Banos, 2019). Alors comment les propriétaires forestiers réagissent-ils face à ces crises ? Pour certains observateurs, les ECE seraient un des principaux moteurs du changement de pratiques forestières (Keskitalo et al., 2015). Les crises offriraient l'occasion d'explorer de nouveaux modes de gestion et de faire preuve d'innovation (Lawrence, 2017). De manière plus préventive, l'aversion aux risques inciterait également les propriétaires forestiers à s'engager dans une démarche réflexive et à adopter de nouveaux comportements (Andersson et Gong, 2010). A contrario, d'autres études montrent qu'après le choc initial, les propriétaires reviendraient progressivement à des pratiques de gestion classique (Lidskog et Sjödin, 2014). Perçu comme un moyen d'atténuer le traumatisme de la catastrophe, ce retour à une gestion routinisée révélerait 
aussi une forme de conservatisme propre à certaines catégories de propriétaires peu enclins au changement et souvent identifiés comme des propriétaires «traditionnels », « suiveurs", voire "passifs» (Blanco et al., 2015). Finalement, dans le domaine forestier aussi, la question n'est pas tant de savoir si les pratiques changent ou non, mais comment, avec quelle intensité et quels facteurs orientent ces changements ou ce retour aux routines.

\section{Méthode}

Étudier l'influence d'un ECE sur des pratiques routinières suppose de décaler le regard par rapport aux moments de tensions (secours, gestion de crise...) qui cristallisent généralement l'attention et de s'intéresser aux effets à plus long terme (Clavandier, 2015). Le matériau principal est ainsi constitué d'enquêtes réalisées près de dix ans après les catastrophes. Si ce délai permet de prendre du recul par rapport aux promesses de changement faites à «chaud», il est cependant intéressant de comprendre comment le problème a été « refroidi ». Cela suppose de retracer l'évolution de l'état d'esprit des propriétaires, les différentes options envisagées et l'institutionnalisation plus ou moins forte des sentiers empruntés. Cette lecture diachronique s'est appuyée sur le témoignage de propriétaires forestiers, de gestionnaires et de représentants institutionnels ayant souvent vécu l'ensemble du processus ainsi que sur des documents d'expertises, des guides techniques et des articles de presse produits à différentes étapes de la crise. Dans un des deux cas étudiés, cette analyse fut enrichie par la remobilisation d'entretiens réalisés au lendemain de la catastrophe. La mise en regard de deux ECE permet aussi d'atténuer les risques de particularismes liés au caractère toujours situé des catastrophes. Elle offre également la possibilité d'isoler quelques différences structurelles (type de sylviculture, organisation de la filière...) et de tester leurs influences sur les mécanismes de décision des propriétaires.

Puisqu'un tournant éventuel n'a de sens que par rapport à un point de référence (Abbott, 2001), il nous faut présenter la situation à la veille de ces deux catastrophes.

Plus grande forêt privée d'Europe occidentale, le massif des Landes de Gascogne est le siège d'une importante filière bois-papier dominée par les industries de la trituration (papetiers, panneautiers). D'un point de vue sylvicole, le modèle landais se caractérise par une gestion dynamique de peuplements monospécifiques de pin maritime avec des rotations standards de 45-55 ans et un recours à la fertilisation, à la mécanisation et à l'amélioration génétique. Sans être exclusif, ce modèle productiviste est très largement diffusé auprès des propriétaires landais privés ( $92 \%$ de la surface). C'est dans ce contexte que, la nuit du 24 janvier 2009, la tempête Klaus renverse 223000 ha de pin maritime, soit $20 \%$ du massif forestier (Fig. 1). Si on y ajoute les dégâts occasionnés par la tempête de 1999 et les attaques phytosanitaires, c'est près de $50 \%$ du capital sur pied qui est tombé en dix ans (Mora et al., 2012). En 2013, nous avons réalisé une campagne d'entretiens auprès de propriétaires forestiers $(n=36)$ et d'acteurs de la filière $(\mathrm{n}=18)$, puis une enquête quantitative ciblée sur les propriétaires en $2015(n=432)$. Cette étude fut étayée par l'analyse des documents techniques et médiatiques rédigés durant la crise et par la reprise d'une enquête qualitative réalisée dans les 2 à 6 mois qui ont suivi la tempête Klaus. Prévue avant la catastrophe et initialement centrée sur une autre problématique, cette enquête avait finalement permis de recueillir les impressions et intentions à chaud d'une quarantaine de propriétaires forestiers (Deuffic et Moustié, 2010).

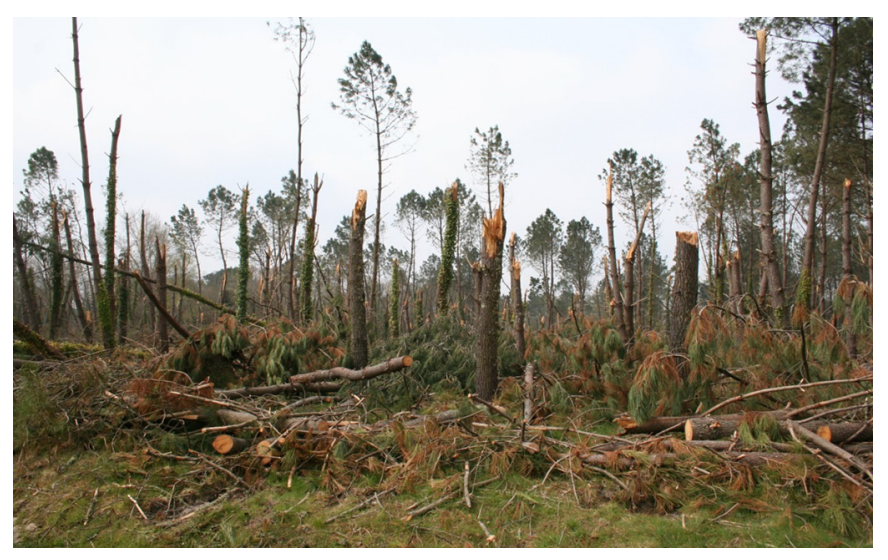

Fig. 1. La tempête Klaus du 24 janvier 2009, un événement climatique extrême dévastateur (@) P. Deuffic, 2009).

Le second cas d'étude est le pays de Sault, petit plateau de 68000 ha boisés à $40 \%$ de sapins, situé dans les Pyrénées audoises. Affectée par la diminution continue du nombre de scieries, la filière locale y est fragile. Le poids relatif des propriétaires privés ( $44 \%$ de la surface), leur faible capacité de représentation et le type de sylviculture pratiquée sont trois autres facteurs de distinction avec les Landes. Plus extensive, la gestion forestière est dominée par des futaies irrégulières de sapins conduites en régénération naturelle sur des rotations de 120 ans et plus. En 2003, cette zone méridionale subit de plein fouet la canicule et une sécheresse sévère se prolonge jusqu'en 2007 (Fig. 2). Au final, près de 5500 ha de sapins sont atteints et $93000 \mathrm{~m}^{3}$ de bois sont perdus. En complément du matériau textuel recueilli, des entretiens $(n=41)$ ont été réalisés en 2017 auprès de propriétaires et de gestionnaires présents au début de la crise pour comprendre comment ils avaient vécu ce phénomène au long cours et comment ils s'étaient adaptés. 


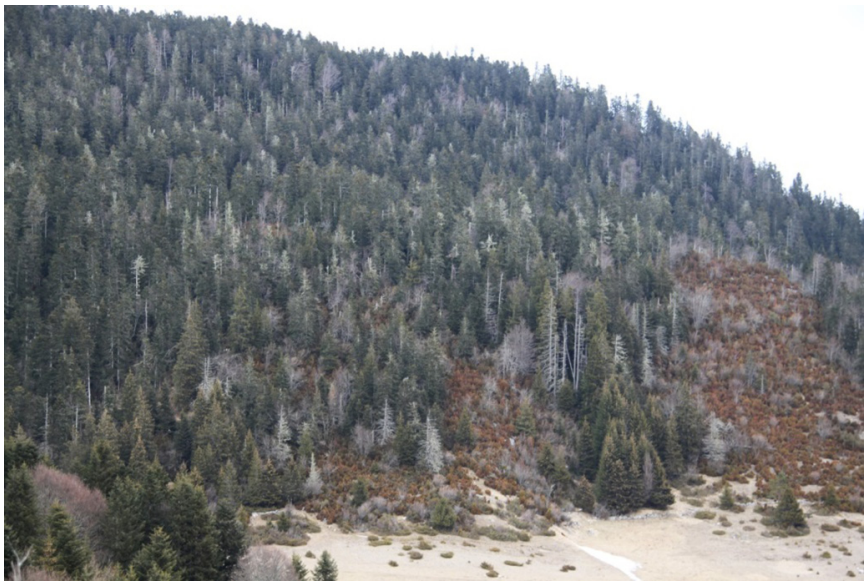

Fig. 2. Le dépérissement des sapins, un phénomène insidieux (C) P. Deuffic, 2015).

\section{Le temps de la crise}

Pour saisir les régimes d'épreuves (Chateauraynaud et Torny, 2013) traversés par les propriétaires forestiers du pays de Sault et des Landes, il faut revenir sur les crises ouvertes par les ECE de 2003 et de 2009. Comment ont-ils réagi face à ces catastrophes ? Où les a conduits la recherche de causes et de solutions à ces événements climatiques?

\section{Le temps des doutes et des questionnements}

Dans le massif landais, malgré la violence du choc (Fig. 1), les propriétaires forestiers font preuve d'une grande réactivité. Ayant encore en tête la tempête de 1999, ils cherchent à vendre rapidement leur bois malgré les mots d'ordre syndicaux. L'urgence est motivée par la peur des attaques phytosanitaires, des risques d'incendie et d'une chute des prix. Mais, contrairement à 1999, le marché est saturé. La crise économique s'ajoutant au volume de bois détruit, les ventes sont réalisées à perte. Les propriétaires vivent aussi très mal les atermoiements de l'État et des pouvoirs publics quant à la nature, au montant et au calendrier des aides demandées (RibereauGayon, 2011). Enfin, l'ampleur des dégâts et la répétition en l'espace d'à peine dix ans d'un événement qualifié en 1999 de «tempête du siècle» suscitent de nombreuses interrogations. À quoi bon faire de la sylviculture si de telles catastrophes se reproduisent fréquemment? Partagé par nombre de propriétaires, ce sentiment d'impuissance est exacerbé par la nature même de l'aléa: "Que faire contre des rafales à $172 \mathrm{~km} / \mathrm{h}$ ? Aucun arbre ne peut résister» (Pierre, responsable syndical forestier, 53 ans). Le hasard et la fatalité s'avèrent pourtant des coupables rapidement insatisfaisants. Pour repartir, ces praticiens ont besoin d'explications rationnelles quitte à remettre en cause les référentiels d'actions éprouvés. Opérant un examen critique de la ligniculture développée depuis les années 1960, des forestiers incriminent alors l'amélioration génétique du pin maritime, le labour, la fertilisation ou encore l'intensité des éclaircies. Malgré les incertitudes et les controverses sur le rôle effectif de ces facteurs, rares sont les propriétaires qui, en 2009, imaginent un retour à l'identique. Les modifications envisagées vont de l'abandon de l'entretien par rouleau landais, accusé de casser le système racinaire, à l'introduction de nouvelles essences feuillues censées mieux résister à la tempête, voire à l'irrégularisation de la futaie de pin maritime (Deuffic et Moustié, 2010).

Dans le pays de Sault, si les forestiers gardent un vif souvenir de la sécheresse de 2003, ils témoignent d'un événement moins brutal et plus étalé dans le temps. De fait, les premiers signes du dépérissement n'apparaissent qu'au printemps 2004. Les sapins sèchent sur pied de façon hétérogène et, à la différence du massif landais, les dégâts sont dispersés (Fig. 2). Cette information sériée et séquentielle retarde le déclenchement de l'alerte. Et ce d'autant plus que, sur ce territoire confronté régulièrement à des sécheresses de basse intensité, la mortalité des sapins constitue un bruit de fond auquel les acteurs sont habitués. Mais, au printemps 2004, les cas de mortalités explosent. Parant au plus pressé, un groupe informel de techniciens du Centre régional de la propriété forestière (CRPF), de coopératives, de l'Office national des forêts (ONF) et d'experts forestiers préconise de procéder à des coupes sanitaires. Cette stratégie est massivement suivie par les propriétaires qui, ici aussi, souhaitent avant tout sauvegarder la valeur des arbres atteints. Cédant de leur propre aveu à un moment de panique, ils coupent alors plus de bois que de raison, ce qui entraîne un engorgement du marché et une chute des prix. En baissant la densité d'arbres à l'hectare, les coupes anticipées diminuent aussi l'ambiance forestière et favorisent la spirale du desséchement. Le désarroi des propriétaires est d'autant plus grand que les pouvoirs publics peinent à prendre la mesure de cet événement localisé et particulièrement insidieux. Ainsi, alors que la crise s'arrête en 2008 avec le retour de pluviométries normales, il n'y a toujours pas de plan d'intervention massif. À l'heure de s'atteler à la reconstitution des peuplements, les propriétaires disposent donc de peu d'informations et de peu de moyens. Désireux de réagir, certains envisagent néanmoins de ne pas reboiser les stations les plus sèches, de modifier les régimes d'éclaircies, de raccourcir l'âge d'exploitation des sapins, voire de changer d'essence (cèdre, hêtre). Comme dans les Landes de Gascogne, l'univers des possibles semble donc s'ouvrir. 


\section{Le temps de la raison et des experts}

Dans les Landes, alors que les propriétaires sont encore submergés par le doute et la colère, les professionnels de la filière et les représentants syndicaux se positionnent rapidement pour mettre à l'agenda politique la question de la reconstitution du massif forestier. Malgré leurs désaccords récurrents, ces acteurs de l'amont et de l'aval, qui se connaissent bien et se côtoient régulièrement dans les arènes sectorielles et régionales, voire nationales, partagent le même souci d'un maintien de l'existant. Ils empruntent alors les chemins classiques de la mise en problème public (Cefaï et Terzi, 2012): objectivations des dégâts, dénonciation médiatique de la passivité des pouvoirs publics, manifestations pour interpeller le public et gagner son soutien, intense lobbying institutionnel. Dramatisant les menaces qui pèsent sur la filière et le territoire ${ }^{1}$, ce travail politique aboutit à l'élaboration, dès février 2009, d'un plan de solidarité nationale dit plan Chablis ${ }^{2}$. Abondé par l'État à hauteur de 475 millions d'euros, ce dispositif conséquent et décidé en urgence contribue à cadrer fortement les trois expertises lancées sur le devenir de la forêt landaise. Il ne s'agit plus tellement de débattre de la pertinence ou non d'une reconstitution, mais bien d'accélérer les reboisements (Laffite et Lerat, 2009). Ainsi, sur les six itinéraires sylvicoles proposés à l'été 2009 par les experts nationaux et régionaux, cinq sont à base de pin maritime (Lesgourgues et Chantre, 2009). L'heure n'est donc pas vraiment à la révolution. Toutefois, trois de ces itinéraires abaissent l'âge standard des peuplements à $35-45$ ans, voire 25 ans, là où les orientations forestières régionales préconisaient encore, en 2001 , des rotations à $55-65$ ans pour les itinéraires «haute qualité ». Si cette réduction de l'âge d'exploitabilité des arbres conforte les trajectoires industrielles engagées depuis plusieurs années (papier, panneaux...), elle constitue une rupture pour les propriétaires landais. Malgré le déclin du marché des gros bois, ces derniers restent attachés à ce type d'arbre, symbole de leur savoirfaire sylvicole et de la dimension patrimoniale de leur propriété (Deuffic et al., 2010). Par ailleurs, si les expertises tendent à entériner l'absence d'alternative économique au pin maritime dans les Landes (Laffite et Lerat, 2009), le plan Chablis n'exclut pas le recours aux feuillus pour reboiser. Il prévoit aussi une clause de diversification destinée à favoriser la biodiversité via notamment la gestion et l'extension des formations feuillues existantes sur les parcelles.

\footnotetext{
${ }^{1}$ Les craintes d'un changement d'usages des sols forestiers et d'une pénurie de bois «à venir» sont associées à des pertes d'emplois et à une remise en cause de l'identité du territoire.

${ }^{2}$ En forêt, les chablis désignent un ensemble d'arbres renversés et déracinés ainsi que les perturbations économiques et écologiques associées.
}

Dans le pays de Sault, la question du dépérissement reste un problème secondaire dont la mise à l'agenda politique ne dépasse guère l'échelle départementale. Face aux dégâts massifs causés par la sécheresse dans les forêts du Massif central, certains experts peinent à qualifier de "catastrophe » le dépérissement de sapins situés dans les Pyrénées audoises, en limite sud de leur aire de répartition (Larminat, 2018). Ne bénéficiant d'aucune subvention directe pour reconstituer leurs peuplements, les propriétaires peuvent cependant s'appuyer sur un programme d'expérimentation (projet Interreg ForClimaAdapt) lancé en 2010 et auquel participe le groupe informel d'experts locaux créé au moment de la crise. Ce programme confirme certaines causes du dépérissement sans pour autant éliminer toutes les incertitudes. La cartographie des dépérissements montre ainsi que les forestiers ont laissé le sapin s'installer sur des zones beaucoup trop sèches. En revanche, les essais visant à augmenter le nombre d'éclaircies ou à éradiquer les buis en sous-bois afin de limiter la compétition pour l'accès à l'eau s'avèrent peu concluants. Cette phase de diagnostic laissant encore beaucoup de questions en suspens, le groupe de travail se replie alors sur des options dans l'air du temps telles que la «gestion dynamique» des peuplements. Les experts préconisent ainsi de raccourcir les rotations du sapin de 140 à 100 ans (CRPF Languedoc Roussillon, 2016). Dans les zones où les régénérations du sapin s'avéreraient vraiment impossibles, ils recommandent d'augmenter la proportion de hêtre, voire d'introduire le cèdre de l'Atlas, une espèce exotique capable de fournir une bonne production de bois sous un climat relativement sec.

Même si les tâtonnements à l'œuvre dans le pays de Sault contrastent avec l'empressement de la filière à restaurer l'unité menacée du collectif dans les Landes de Gascogne, le processus de sortie de crise tend, dans les deux cas, à conforter l'autorité des acteurs "en place», qu'ils soient conseillers ou agents de développement forestier publics et privés, entrepreneurs de travaux sylvicoles, représentants des industries du bois et des services déconcentrés forestiers de l'État. Sollicités en tant qu'experts, ils ont rapidement restreint le champ des débats et privilégié des options d'adaptation déjà discutées et expérimentées. Ce faisant, ils ont permis de passer d'une situation d'indétermination à l'issue imprévisible à une analyse de risques replaçant au centre des débats les rationalités et les calculs technicoscientifiques habituels. L'idée générale est ainsi d'améliorer la résistance du système en misant sur l'intensification et la dynamisation des itinéraires existants (raccourcissement des rotations et introduction d'essences productives à croissance rapide). Néanmoins, quelques options alternatives davantage basées sur la résilience que sur l'optimisation de la productivité 
ligneuse (clause diversification, revalorisation du hêtre) subsistent à l'issue des expertises. De plus, à la différence des sylviculteurs landais qui doivent se plier aux normes techniques du plan Chablis, les propriétaires du pays de Sault ne sont pas liés contractuellement aux décisions des experts car ils ne disposent d'aucune aide financière pour appliquer les recommandations. À l'issue de la crise, on constate ainsi que si les ECE n'ont pas renversé la table, ils ont permis d'instiller des germes de changements. Reste à savoir ce qu'il en reste dix ans après.

\section{Dix ans après, des bifurcations modestes}

Vu que les stratégies d'adaptation esquissées oscillent entre retour aux routines et intensification des pratiques sylvicoles, on peut s'interroger sur les facteurs de décisions des propriétaires et sur l'influence réelle des ECE, ou du moins du recadrage qu'en ont proposé les experts.

\section{Le retour aux routines}

Presque dix ans après la tempête Klaus, le risque d'un changement d'usages des sols forestiers, un temps agité par les acteurs de la filière, ne s'est pas réalisé. Sur les 204000 ha éligibles, 198000 sont nettoyés et 192000 sont prévus à la plantation ${ }^{3}$. Quant aux pistes visant à introduire une plus grande diversité d'essences, elles ont disparu des options choisies par les propriétaires puisque $95 \%$ des reboisements sont réalisés en pin maritime. Les rares cas de diversification concernent des essences de production comme le robinier, le pin taeda et l'eucalyptus. Quant à la clause «biodiversité» censée s'appliquer jusqu'à $30 \%$ des surfaces détruites, elle n'a finalement été mise en œuvre que sur $6 \%$ des surfaces éligibles. Pour justifier ce qui s'apparente à une reconstitution à l'identique, beaucoup d'enquêtés reprennent l'argumentation des experts sur les conditions stationnelles du massif ou la configuration du tissu industriel local, qui rendraient illusoire toute alternative au pin maritime. Ils évoquent aussi leur attachement aux critères de performance classiques du modèle landais - productivité plutôt que résilience. À cela s'ajoute un manque de recul vis-à-vis des nouveaux itinéraires qui incite à la prudence et qui ne pousse à guère plus que des expérimentations, «juste pour voir». Enfin, même si les coûts de reboisement sont couverts par le plan Chablis, la tempête a réduit la flexibilité économique des propriétaires en les obligeant à anticiper des investissements

\footnotetext{
${ }^{3}$ Relevé d'informations du comité tempête de la direction régionale de l'Alimentation, de l'Agriculture et de la Forêt (DRAAF) Aquitaine (16 février 2017).
}

qu'ils n'avaient pas forcément prévu de faire avant 10 ou 20 ans.

Dans le pays de Sault, la même prudence prévaut mais pour des raisons différentes. Les propriétaires rappellent ainsi que des préconisations techniques (coupes anticipées, augmentation des éclaircies, éradication du buis) émises pendant la sécheresse se sont révélées après coup de fausses bonnes idées. S'ils n'éprouvent pas de regret vis-à-vis de ces essais, estimant que l'expérimentation et le droit à l'erreur font partie de leur activité, les propriétaires n'ont généralement pas étendu ces mesures. L'heure est aussi à la temporisation en ce qui concerne la fin de règne du sapin. Pourtant, condamné par certains experts nationaux qui considèrent le changement inéluctable à plus ou moins long terme, le roi des forêts du pays de Sault bénéficie encore localement d'un sursis faute d'alternatives convaincantes. Les propriétaires ont, en effet, des doutes sur l'avenir économique du hêtre au regard de la demande industrielle actuellement centrée sur les résineux. Quant au cèdre de l'Atlas, il doit encore faire ses preuves. Nécessitant un investissement conséquent, de l'ordre de 5000 euros/ha, sa plantation est circonscrite aux zones où la régénération de sapins est impossible. Et, en l'absence de subventions, seuls les propriétaires les mieux dotés en capital financier se permettent cette prise de risque. Au-delà des enjeux de rentabilité économique, les propriétaires s'interrogent aussi sur l'adaptation de cette essence exotique aux conditions locales, sur sa sensibilité supposée aux dégâts de gibiers ou encore ses effets sur la biodiversité et la fertilité des sols. Ces zones d'ombre ne favorisent pas l'adoption de cette essence de substitution. In fine, les propriétaires se rangent à l'avis des experts locaux qui, tout en conseillant de limiter son aire d'extension, réaffirment que le «sapin a de l'avenir » (CRPF Languedoc Roussillon, 2016).

\section{Des changements significatifs: entre intensification et abandon de la gestion}

Si les ECE n'ont pas délégitimé les anciennes routines, ils ont contribué à réactiver le débat sur le vieillissement de la forêt et la surcapitalisation sur pied de la ressource (Orazio et al., 2018). En effet, si le raccourcissement des rotations est au fondement même de la sylviculture, les pourfendeurs des peuplements dits « âgés », contenant des " gros » bois, peinaient jusque-là à convaincre les propriétaires de renoncer à ces arbres. Même l'idée d'un marché de plus en plus orienté vers les petits et les moyens bois n'y suffisait pas. Cette demande apparaissait trop pilotée par des préoccupations industrielles divergentes de leurs propres intérêts. Or, les ECE ont permis d'ajouter un nouveau cadre interprétatif auquel les propriétaires se sont montrés sensibles: en plus de rajeunir la forêt et de répondre aux «vrais» 
besoins du marché, réduire l'âge d'exploitation des bois permettrait d'éviter la surexposition du capital sur pied aux risques climatiques. L'enquête menée en 2016 (Brahic et Deuffic, 2017) confirme cet alignement des motifs d'action : $39 \%$ des propriétaires landais envisagent comme premier choix d'itinéraire des rotations à $35-45$ ans; $14 \%$ envisagent même des rotations inférieures à 35 ans, ce que personne ne faisait avant la tempête. Dans le pays de Sault, la même tendance se profile puisque les enquêtés se conforment peu à peu aux préconisations des experts locaux en raccourcissant les rotations de 140 à 100 ans. Conséquente et assez spectaculaire, cette adhésion au raccourcissement des cycles de culture n'est pas pour autant totale. Au moins $25 \%$ des propriétaires landais disent encore opter pour des rotations supérieures à 45 ans. Les propriétaires forestiers les plus âgés préfèrent différer les changements de pratiques les plus importants et les déléguer à la génération suivante (Deuffic et al., 2018). Pour d'autres, la crainte d'un ECE qu'ils savent inéluctable à moyen terme ne suffit pas à compenser les risques économiques liés à un engorgement du marché par des produits de faible valeur ajoutée. Ils parient donc sur un rebond des «gros bois» porté par le marché de la construction. Ils redoutent aussi que le terme «dynamique» rime avec intensification et que la maximisation de la croissance soit favorisée aux dépens du maintien des essences d'accompagnement, de la biodiversité ou de la qualité des paysages.

Les ECE ont également induit une évolution des pratiques vis-à-vis du bois énergie (BE). Avant la tempête Klaus, les propriétaires landais étaient circonspects vis-à-vis de ce nouveau marché jugé peu structuré, peu rémunérateur et présentant des risques potentiels pour la biodiversité et la fertilité des sols (Deuffic et Lyser, 2012). Après la tempête, la part de propriétaires ayant vendu du $\mathrm{BE}$ a pourtant bondi, passant de $10 \%$ à $29 \%$ (Brahic et Deuffic, 2017). Si l'adoption de ce nouveau comportement a été facilitée par l'essor des consommations du BE dans le sillage du développement des politiques «Climats-Énergie» (Banos et Dehez, 2015), les ECE ont aussi contribué à «enrôler» les propriétaires. Dans un contexte de crise, le BE apparaît, en effet, comme un moyen de couvrir des besoins de trésorerie à court terme, une possibilité de répartir les risques économiques sur différents types de marché, voire une manière de revaloriser certaines essences feuillues telles que le hêtre dans le pays de Sault. Dans le cas des souches du pin maritime, on peut même parler d'un élargissement significatif du spectre des ressources exploitables (Banos et Dehez, 2017). En incitant les propriétaires à enlever les souches pour nettoyer leurs parcelles, les tempêtes de 1999 et 2009 ont facilité et accéléré l'accès à une ressource désirée par les papetiers depuis la première crise pétrolière mais toujours refusée par les sylviculteurs. L'essai s'avère d'autant plus concluant que les industriels ont trouvé les arguments pour séduire les propriétaires au-delà de la crise : l'extraction des souches permettrait de réduire les coûts de reboisement et les risques phytosanitaires. Au final, malgré des incertitudes persistantes sur le «juste» prix de cette nouvelle ressource et les effets de son prélèvement sur la fertilité des sols et le stockage du carbone, $70 \%$ des propriétaires landais se disent désormais prêts à extraire les souches pour en faire du BE (Brahic et Deuffic, 2017).

Tout en favorisant la diffusion et la banalisation de pratiques sylvicoles intensives, les ECE ont également éloigné, voire sorti définitivement, certains propriétaires du champ de la gestion forestière. Peu médiatisés et publicisés, ces changements n'en sont pas moins significatifs. Dans le pays de Sault, alors que l'attention s'est surtout focalisée sur le sapin, les dépérissements ont aussi affecté des parcelles d'épicéas appartenant souvent à de petits propriétaires. Sans fonds propres ni aides publiques pour reboiser, ces forestiers sont les perdants quasi invisibles de la sécheresse de 2003 et leurs parcelles sont aujourd'hui à l'abandon. De même, dans les Landes de Gascogne, les propriétaires de 1 à 4 ha n'étaient initialement pas éligibles au plan Chablis. II aura fallu la mobilisation des représentants syndicaux et des élus locaux pour que leur cas soit reconsidéré. Mais faiblement doté en capital économique et peu inséré dans les réseaux professionnels, ce type de propriétaire n'est pas le plus enclin à prendre des risques. Pour certains d'entre eux, les ECE ont donc signé l'arrêt définitif de leur investissement dans la sylviculture.

\section{Discussion}

Ni les ravages causés par la répétition de deux tempêtes majeures à dix ans d'intervalle ni les dépérissements massifs induits par la sécheresse historique de 2003 n'ont réussi à ébranler en profondeur les certitudes et à bouleverser la gestion forestière dans les Landes et dans les Pyrénées audoises. Ce résultat peut être source de déception car "l'illusion héroïque» (Dobry, 2010) conduit intuitivement à attendre beaucoup d'événements considérés comme extraordinaires. Mais la remarquable stabilité de ces systèmes sociotechniques est aussi quelque peu trompeuse car la continuité observée n'est pas dénuée de changements. Formant des «conjonctures fluides» (Dobry, 2010), les catastrophes ont en effet suspendu les segmentations sectorielles qui prévalaient entre l'amont et l'aval de la filière, contribué à ouvrir celle-ci à de nouveaux enjeux et de nouveaux acteurs (l'énergie) et permis un travail de délégitimation des habitudes. Des changements de pratiques, que des années de sensibilisation n'étaient 
pas parvenues à impulser, l'ont ainsi été grâce aux fenêtres d'opportunité qu'ont constituées ces deux ECE. $\mathrm{Au}$ final, ces derniers ont conduit les propriétaires à opérer une forme de "bifurcation assistée », et souvent par défaut, de leurs itinéraires sylvicoles.

Une des explications de ce changement dans la continuité tient en effet à l'autorité épistémique conférée par les propriétaires aux experts, c'est-à-dire à leur légitimité à décrire et à expliquer la réalité (Gieryn, 1999). Cette forme d'autorité leur a permis de cadrer l'événement, ses causes et les solutions envisageables, tout en minimisant ou en rendant invisibles d'autres interprétations. Un temps remis en cause par quelques acteurs en marge du système, les porteurs de cette forme d'autorité ont bénéficié d'un accès privilégié aux arènes de discussion et de légitimation (Gilbert et Henry, 2012) qu'ont constituées les expertises mises en place après les deux ECE. Le fait qu'ils soient souvent les seules personnes-ressources disponibles, voire invitées, leur a aussi permis de naturaliser, redresser, voire refermer des trajectoires déjà pensées par ailleurs, comme cela est le cas pour les six options proposées par le groupe de travail « Itinéraires sylvicoles » dans les Landes. La crise n'étant pas un moment propice pour la réflexion sur table ou au bureau (Lidskog et Sjödin, 2014), la plupart des experts avaient déjà anticipé - via les dispositifs de prévention de crise - les différentes façons de gérer ces événements avant qu'ils n'arrivent. Possédant une longueur d'avance, ils ont proposé des solutions prêtes à l'emploi, immédiatement mobilisables et déjà plus ou moins expérimentées, à l'image de l'extraction des souches (Banos et Dehez, 2017) ou des plantations de cèdres. Ils ont laissé entrevoir aux victimes une porte de sortie dans laquelle il était plus facile de s'engouffrer plutôt que de chercher de l'information dans d'autres sphères de connaissance. De fait, ces formes d'objectivation des choix techniques ont fonctionné comme des «illusions» socialement nécessaires (Feenberg, 2004) dans la mesure où elles ont rassuré les propriétaires, même si la viabilité, la robustesse et la résilience de ces options à long terme sont difficiles à évaluer. Mais, sous couvert de rationalité, d'objectivité, voire de "neutralité » et d'intérêt général, ces mesures peinent à dissimuler les intentions de leurs promoteurs qui visent surtout à cadrer le problème et ses solutions en fonction de leurs propres intérêts.

Une autre explication à cette relative inertie tiendrait au conservatisme supposé des propriétaires forestiers qui privilégieraient les comportements routiniers et développeraient une aversion au risque les dissuadant d'effectuer des changements incertains (Brunette et al., 2009). Le comportement humain en général - et les forestiers n'y échappent pas - tend en effet à privilégier les pratiques routinières qui évitent d'avoir à décider trop souvent et assurent à l'individu une sécurité ontologique (Dynes et Drabek, 1994). L'adhésion à un modèle de sylviculture bien établi permet ainsi de gagner du temps, voire de l'optimiser. Pour autant, ce postulat du conservatisme ne résiste pas aux observations de terrain qui montrent une évolution permanente des référentiels de gestion, évolution qui s'accélère parfois brutalement à l'occasion d'une catastrophe. Les propriétaires suivent alors les évolutions techniques proposées par les professionnels de la filière, qu'il s'agisse de raccourcir les rotations ou de planter de nouvelles essences. Comme après la tempête Gudrun en Suède (Lidskog et Sjödin, 2014), des changements de pratiques ont bien eu lieu mais de façon progressive et incrémentale. Les propriétaires landais et pyrénéens ont ainsi opéré des changements sélectifs basés sur des critères de pertinence mêlant à la fois intérêt économique, prises de risque limitées et prise en compte partielle de variables telles que le changement climatique. Alors qu'une gestion plus extensive ou plus proche de la nature aurait représenté un vrai changement de paradigme, le raccourcissement des rotations, l'accélération du régime d'éclaircie et l'exploitation des sousproduits de la sylviculture ne sont finalement que la dernière étape d'un processus d'intensification auquel les propriétaires forestiers ont toujours adhéré jusqu'à présent. Cette gestion dite «dynamique» des forêts s'aligne finalement bien avec leurs cadres d'interprétation et les normes en vigueur en matière de sylviculture. La convergence des arguments naturalise ainsi certaines options sylvicoles et fonctionne comme une entreprise de moralisation (Becker, 1985) des pratiques sylvicoles et des relations entre acteurs de la filière. Les ECE permettent en effet aux tenants de la modernisation sylvicole de dénoncer les pratiques traditionnelles (non-recours à l'amélioration génétique, surcapitalisation des bois sur pied, gestion extensive des peuplements, etc.), d'en stigmatiser les auteurs et de justifier en réaction une réforme des conduites consistant à couper les gros bois et à raccourcir les rotations. D'une certaine manière, l'invisibilisation des perdants de la catastrophe, notamment les petits et très petits propriétaires, s'inscrit également dans cette optique. En contrepoint de la prime au courage décernée aux sylviculteurs qui sont repartis de l'avant, ces exclus de la communauté des forestiers sont en effet oubliés et laissés à leur sort, voire désignés comme coupables de leur propre malheur pour ne pas avoir voulu couper les arbres à temps, et comme incapables de se relever d'eux-mêmes. Lancinante, cette interprétation morale de la catastrophe contribue à éluder la grande disparité économique des propriétaires et leur inégale capabilité à opérer une transition (Nussbaum, 2012).

Si les propriétaires forestiers sont fortement poussés à changer, ils n'adhèrent pas non plus aveuglément aux discours des experts. Leur choix est modulé par la 
confiance dans celui qui porte l'innovation (Gootee et al., 2010), la perception des opportunités et des risques que ces changements sous-tendent (Lidskog et Sjödin, 2014), les objectifs, les valeurs et les logiques d'action qui orientent la gestion forestière (Deuffic et al., 2018). Ils sont ainsi très méfiants vis-à-vis des innovations les plus «révolutionnaires » comme les itinéraires à très courte rotation. Choisir un de ces itinéraires en situation normale est déjà un risque pour un propriétaire. En temps de crise, cela leur paraît encore plus aléatoire. Ce besoin de temps pour opérer des changements structurels se voit notamment dans leur façon de s'approprier les itinéraires dits de «gestion adaptative», méthode qui s'appuie sur l'apprentissage -qu'il provienne du bon sens, de l'expérience, de l'expérimentation, du suivi...- en adaptant les pratiques en fonction de ce qui a été appris (Bormann et al., 1999). Considérant les incertitudes sur la rapidité et la nature des changements climatiques, les propriétaires forestiers parient sur la possibilité de s'y adapter par palier, de procéder par essai erreur, d'évaluer à chaque étape la viabilité de l'itinéraire technique mais aussi sa réversibilité (Grothmann et Patt, 2005). La question de l'adaptation au changement climatique est donc bien une variable intervenant dans le choix d'un modèle de gestion, mais ce n'est pas toujours une priorité. L'extraction des souches procède ainsi d'une opportunité strictement économique (réduction des coûts de reboisement) renforcée par d'autres avantages qui n'ont rien de climatiques (réduction des risques phytosanitaires). Quant à la replantation généralisée de pin maritime, elle relève aussi d'une gestion opportuniste des risques car elle assure à moyen terme, pour les propriétaires, un débouché et, pour les industriels, une sécurité d'approvisionnement. Bien que rassurant, ce retour à des schèmes connus minore, voire occulte, d'autres risques: les sécheresses et les incendies, dont la fréquence et l'ampleur risquent d'augmenter avec le réchauffement climatique. Ce reboisement laisse surtout en suspens la question de l'émergence de nouveaux pathogènes potentiellement dévastateurs comme le nématode du pin qui a un impact sanitaire et économique considérable dans le nord de l'Espagne et dont les modèles de diffusion prévoient l'arrivée en France d'ici 2025 (Robinet et al., 2011).

\section{Conclusion}

La tempête Klaus et la sécheresse de 2003 constituent indéniablement des catastrophes pour les propriétaires forestiers qui les ont subies. Pourtant, comme les spécialistes des disaster studies ou les sociologues de l'événement, nous constatons qu'elles n'entraînent pas forcément de révolution copernicienne. En revanche, elles déclenchent, voire accélèrent, des transformations déjà en germe avant l'événement. En instrumentalisant le sentiment de continuité qui permet de masquer et de naturaliser certaines bifurcations, les décideurs forestiers parviennent finalement à initier des changements de pratiques qui ne demandaient qu'une fenêtre d'opportunité pour s'exprimer (Journet, 2010).

Mais était-il vraiment utile de passer par la catastrophe pour étudier ces changements de pratiques sachant que nous aurions pu nous contenter de mobiliser le paradigme du risque? D'ailleurs, l'analyse menée sur le travail de définition et de mise à l'agenda des crises en tant que problèmes publics emprunte largement à cette littérature. Mais, la principale limite au concept de risque et ses notions afférentes - prévention, prévoyance, principe de précaution, vigilance, vulnérabilité- reste son caractère probabiliste (Clavandier, 2015). Or, les crises ne peuvent plus être seulement perçues comme des horizons; comme le dit Dupuy (2002), «il nous faut apprendre à affronter la catastrophe, à ne plus l'imaginer dans un futur improbable mais à la penser au présent». Dans ce contexte de porosité croissante entre situations de risques et situations de crises (Gilbert, 2013), l'entrée par la catastrophe permet donc de décaler le regard et de mettre à l'épreuve des faits le monde qui nous entoure et les experts qui en parlent. À cet égard, la tempête Klaus et la sécheresse de 2003 auraient dû sérieusement ébranler l'autorité épistémique des experts. Or, celle-ci s'est trouvée renforcée grâce à un accès privilégié aux arènes de débats et à leur statut de seuls référents disponibles, y compris pour expertiser les causes de crises dont ils ont $\mathrm{pu}$, par leurs préconisations antérieures, être en partie responsables. Alors qu'il est désormais beaucoup question de concertation et de coproduction des expertises pour une meilleure gestion des risques, ce type de résultat invite à ne pas oublier le fait que certains acteurs, y compris dans des situations critiques, peuvent «jouer la crise» pour défendre des positions ou en gagner d'autres en stigmatisant des pratiques et en faisant lever des frontières intra- ou extra- sectorielles (Gilbert, 2013; Dobry, 1983).

Étudier les changements de pratiques à l'occasion d'une catastrophe permet aussi de questionner les temporalités et les rythmes du changement (Lidskog et Sjödin, 2014; Nobert et al., 2017) ainsi que le rôle respectif des facteurs structurels ou conjoncturels dans la prise de décision finale de l'individu. Ainsi, si le poids des structures et des normes reste déterminant dans le monde forestier, celles-ci sont suffisamment ébranlées pour que leurs promoteurs décident de les adapter à plus ou moins court terme. Subir la catastrophe accélère donc l'adaptation au prochain événement du même ordre. En effet, à la différence des risques technologiques majeurs dont on peut plus ou moins limiter la récurrence et la sévérité, les ECE sont amenés à se répéter et même à s'aggraver sans qu'on puisse vraiment l'empêcher pour l'instant. Cela induit un changement de posture dans 
l'adaptation des forêts au changement climatique qui ne doit plus viser seulement la prévention d'un risque climatique putatif mais se préparer d'ores et déjà à la prochaine catastrophe. De ce point de vue, là aussi, il y a une réelle plus-value à étudier les catastrophes. Elles constituent des laboratoires et des révélateurs en condition réelle de la pertinence et de l'efficacité des stratégies dites adaptatives, dont Birkland (2009) disait déjà qu'elles ne constituaient parfois rien d'autre que des «fantasy documents» permettant à leurs auteurs - souvent des décideurs publics - de se dédouaner de toute future responsabilité à la prochaine catastrophe venue.

\section{Références}

Abbott A.D., 2001. Time matters. On theory and method, Chigago, The University of Chicago Press.

Andersson M., Gong P., 2010. Risk preferences, risk perceptions and timber harvest decisions-an empirical study of nonindustrial private forest owners in northern Sweden, Forest Policy and Economics, 12, 5, 330-339, http://dx.doi.org/10.1016/j.forpol.2010.02.002.

Banos V., Dehez J., 2015. Les trajectoires du bois-énergie en Aquitaine: du développement local aux territoires de l'énergie ?, Géocarrefour, 90, 4, 329-338, http://dx.doi.org/ 10.4000/geocarrefour.9947.

Banos V., Dehez J., 2017. Le bois-énergie dans la tempête, entre innovation et captation? Les nouvelles ressources de la forêt landaise, Natures Sciences Sociétés, 25, 2, 122-133, http://dx.doi.org/10.1051/nss/2017024.

Bates F., Peacock W., 1987. Disaster and social change, in Dynes R.R., De Marchi B., Pelanda C. (Eds), Sociology of disasters, Milan, Franco Angeli, 291-330.

Becker H.S., 1985. Outsiders. Études de sociologie de la déviance, Paris, Éditions Métailié.

Bessin M., Bidart C., Grossetti M. (Eds), 2010. Bifurcations. Les sciences sociales face aux ruptures et à l'événement, Paris, La Découverte.

Bensa A., Fassin E., 2002. Les sciences sociales face à l'événement, Terrain, 38, 5-20, http://dx.doi.org/10.4000/ terrain. 1888.

Birkland T.A., 2009. Disasters, lessons learned and fantasy documents, Journal of Contingencies and Crisis Management, 17, 3, 146-156, http://dx.doi.org/10.1111/ j.1468-5973.2009.00575.x.

Blanco V., Brown C., Rounsevell M., 2015. Characterising forest owners through their objectives, attributes and management strategies, European Journal of Forest Research, 134, 6, 1027-1041, http://dx.doi.org/10.1007/ s10342-015-0907-x.

Bormann B.T., Martin J.R., Wagner F.H., Wood G., Alegria J., Cunningham P.G., Brookes M.H., Friesema P., Berg J., Henshaw J., 1999. Adaptive management, in Johnson N.L., Malk A.J, Szaro R.C., Sexton W.T. (Eds), Ecological stewardship. A common reference for ecosystem management, Kidlington, Elsevier Science, 505-533.
Brahic E., Deuffic P., 2017. Comportement des propriétaires forestiers landais vis-à-vis du bois énergie: une analyse micro-économique, Économie rurale, 359, 7-25, http://dx. doi.org/10.4000/economierurale.5176.

Brunette M., Cabantous L., Couture S., Stenger A., 2009. Assurance, intervention publique et ambiguïté : une étude expérimentale auprès de propriétaires forestiers privés, Économie et Prévision, 190-191, 4-5, 123-134, www. persee.fr/doc/ecop_0249-4744_2009_num_190_4_8000.

Cabane L., 2012. Gouverner les catastrophes. Politiques, savoirs et organisation de la gestion des catastrophes en Afrique $d u$ Sud. Thèse de doctorat en sociologie, Paris, Sciences Po Paris.

Cabane L., Revet S., 2015. La cause des catastrophes. Concurrences scientifiques et actions politiques dans un monde transnational, Politix, 111, 3, 47-67, http://dx.doi. org/10.3917/pox.111.0047.

Carr L.J., 1932. Disaster and the sequence-pattern concept of social change, American Journal of Sociology, 38, 2, $207-$ 218, http://dx.doi.org/10.1086/216030.

Cefaï D., Terzi C. (Eds), 2012. L'expérience des problèmes publics. Perspectives pragmatistes, Paris, Éditions de l'EHESS.

Chateauraynaud F., Torny D., 2013 [1 ${ }^{\text {re }}$ éd. 1999]. Les Sombres précurseurs. Une sociologie pragmatique de l'alerte et du risque, Paris, Éditions de l'EHESS.

Clavandier G., 2015. Un retour de la catastrophe sur la scène scientifique? Enjeux et débats, Communications, 96, 1, 93105, http://dx.doi.org/10.3917/commu.096.0093.

Coninck F. de, Godard F., 1990. L'approche biographique à l'épreuve de l'interprétation. Les formes temporelles de la causalité. Revue française de sociologie, 31, 1, 23-53.

CRPF (Centre régional de la propriété forestière) Languedoc Roussillon, 2016. Sapinières du pays de Sault et dépérissements : savoir adapter sa gestion, Montpellier, CRPF.

Decocq G., Kalaora B., Vlassopoulos C., 2016. La forêt salvatrice. Reboisement, société et catastrophe au prisme de l'histoire, Seyssel, Champ Vallon.

Deuffic P., Banos V., 2019. Éprouver et s'adapter au changement climatique. Les forestiers landais et pyrénéens entre prudence, résistance, et résilience, Ethnographiques. org, 38, http://dx.doi.org/10.25667/ethnographiques/ 2019-38/006.

Deuffic P., Sotirov M., Arts B., 2018. "Your policy, my rationale". How individual and structural drivers influence European forest owners' decisions, Land Use Policy, 79, 1024-1038, http://dx.doi.org/10.1016/j.landuse pol.2016.09.021.

Deuffic P., Lyser S., 2012. Biodiversity or bioenergy: is deadwood conservation an environmental issue for French forest owners?, Canadian Journal of Forest Research, 42, 8, 1491-1502, http://dx.doi.org/10.1139/x2012-073.

Deuffic P., Ginelli L., Petit K., 2010. Patrimoine foncier... et naturel ? Les propriétaires forestiers face à l'écologisation des Landes de Gascogne, Sud-Ouest Européen, 30, 109124, http://dx.doi.org/10.4000/soe.1296.

Deuffic P., Moustié J., 2010. Pins et feuillus, entre doutes et incertitudes. Les forestiers des Landes de Gascogne et la 
question de la multifonctionnalité des boisements feuillus après la tempête de 2009. Rapport réalisé pour le Conseil régional d'Aquitaine, Cestas, Irstea (version disponible sur Hal-INRAE, https://hal.inrae.fr//hal-02593809/docu ment).

Dobry M., 1983. Mobilisations multisectorielles et dynamique des crises politiques: un point de vue heuristique, Revue française de sociologie, 24, 3, 395-419, www.persee.fr/doc/ rfsoc_0035-2969_1983_num_24_3_3672.

Dobry $\bar{M}$., 2010. La politique dans ses états critiques : retour sur quelques aspects de l'hypothèse de continuité, in Bessin M., Bidart C., Grossetti M. (Eds), Bifurcations. Les sciences sociales face aux ruptures et à l'événement, Paris, La Découverte, 64-89.

Dodier N., 2013. Post-face: Penser (par) la catastrophe, in Revet S., Langumier J., Le gouvernement des catastrophes, Paris, Karthala, 251-275.

Dupuy J.-P., 2002. Pour un catastrophisme éclairé. Quand l'impossible est certain, Paris, Le Seuil.

Dynes R.R., Drabek T.-E., 1994. The structure of disaster research: its policy and disciplinary implications, International Journal of Mass Emergencies and Disasters, 12, 1, 5-23.

Feenberg A., 2004. (Re)penser la technique. Vers une technologie démocratique, Paris, La Découverte.

Geels F.W., Schot J., 2007. Typology of sociotechnical transition pathways, Research Policy, 36, 3, 399-417, http:// dx.doi.org/10.1016/j.respol.2007.01.003.

Gieryn T.F., 1999. Cultural boundaries of science. Credibility on the line, Chicago, University of Chicago Press.

Gilbert C., 1992. Le pouvoir en situation extrême: catastrophes et politique, Paris, L'Harmattan.

Gilbert C., Henry E., 2012. La définition des problèmes publics: entre publicité et discrétion, Revue française de sociologie, 53, 1, 35-59, http://dx.doi.org/10.3917/ rfs.531.0035.

Gilbert C., 2013. Quels risques pour la recherche en sciences humaines et sociales ?, in Bourg D., Joly P.-B., Kaufman A., Du risque à la menace. Penser la Catastrophe, Paris, Presses universitaires de France, 219-235.

Gootee R.S., Blatner K.A., Baumgartner D.M., Carroll M.S., Weber E.P., 2010. Choosing what to believe about forests: differences between professional and non-professional evaluative criteria, Small-scale Forestry, 9, 2, 137-152, http://dx.doi.org/10.1007/s11842-010-9113-3.

Grossetti M., 2006. L'imprévisibilité dans les parcours sociaux, Cahiers internationaux de sociologie, 120, 1, 528, http://dx.doi.org/10.3917/cis.120.0005.

Grothmann T., Patt A., 2005. Adaptive capacity and human cognition: the process of individual adaptation to climate change, Global Environmental Change, 15, 199-213.

Henry J., 2011. Continuity, social change and Katrina, Disasters, 35, 1, 220-242, http://dx.doi.org/10.1111/ j.1467-7717.2010.01201.x.

Journet N., 2010. Catastrophes et ordre du monde, Terrain, 54, 4-10, https://journals.openedition.org/terrain/13916.
Keskitalo E.C.H., Legay M., Marchetti M., Nocentini S., Spathelf P., 2015. The role of forestry in national climate change adaptation policy: cases from Sweden, Germany, France and Italy, International Forestry Review, 17, 1, 3042, http://dx.doi.org/10.1505/146554815814725068.

Kingdon J.W., 1995 [1 ${ }^{\text {st }}$ ed. 1984]. Agendas, alternatives, and public policies, New York, Harper Collins.

Laffite J.-J., Lerat J.-F., 2009. Reconstitution des peuplements forestiers détruits par la tempête du 24 janvier 2009 dans le massif forestier des Landes de Gascogne, Paris, Conseil général de l'agriculture, de l'alimentation et des espaces ruraux, www.vie-publique.fr/sites/default/files/rapport/pdf/ 094000617.pdf.

Larminat J. de, 2018. Changement climatique, la forêt sans le sapin? Les forestiers face au dépérissement de la sapinière du Pays de Sault. Mémoire de fin d'étude d'ingénieur, Talence, Bordeaux Sciences Agro.

Lawrence A., 2017. Adapting through practice: silviculture, innovation and forest governance for the age of extreme uncertainty, Forest Policy and Economics, 79, 50-60, http:// dx.doi.org/10.1016/j.forpol.2016.07.011.

Lesgourgues Y., Chantre G., 2009. Expertise sur l'avenir du massif des Landes de Gascogne. Rapport de synthèse du groupe de travail «Itinéraires sylvicoles», Bordeaux, Gip ECOFOR, www.yumpu.com/fr/document/read/36762044/ rapport-de-synthase.

Lidskog R., Sjödin D., 2014. Why do forest owners fail to heed warnings? Conflicting risk evaluations made by the Swedish forest agency and forest owners, Scandinavian Journal of Forest Research, 29, 3, 275-282, http://dx.doi. org/10.1080/02827581.2014.910268.

MAAF (ministère de l'Agriculture de l'Agroalimentaire et de la Forêt), 2014. Enquête sur la structure de la forêt privée en 2012, Agreste Chiffres et Données, 222, 1-78.

Markard J., Raven R., Truffer B., 2012. Sustainability transitions: an emerging field of research and its prospects, Research Policy, 41, 6, 955-967, http://dx.doi.org/10.1016/ j.respol.2012.02.013.

Mora O., Banos V., Carnus J.-M., Regolini M. (Eds), 2012. Le massif des Landes de Gascogne à l'horizon 2050. Rapport de l'étude prospective commanditée par le Conseil régional d'Aquitaine et l'INRA, Bordeaux, INRA/Conseil régional d'Aquitaine.

Moreau Y., 2017. Vivre avec les catastrophes, Paris, Presses universitaires de France.

Nobert S., Rebotier J., Vallette C., Bouisset C., Clarimont S., 2017. Resilience for the Anthropocene? Shedding light on the forgotten temporalities shaping post-crisis management in the French Sud Ouest, Resilience, 5, 3, 145-160, http:// dx.doi.org/10.1080/21693293.2016.1241479.

Nussbaum M.C., 2012. Capabilités. Comment créer les conditions d'un monde plus juste?, Paris, Climats.

Orazio C., Kies U., Edwards D.M., 2018. Handbook for wood mobilisation in Europe. Measures for increasing wood supply from sustainably managed forests, Bordeaux, European Forest Institute. 
Quarantelli E.L., Dynes R.R., 1977. Response to social crisis and disaster, Annual Review of Sociology, 3, 23-49, http:// dx.doi.org/10.1146/annurev.so.03.080177.000323.

Revet S., Langumier J., 2013. Le gouvernement des catastrophes, Paris, Karthala.

Ribereau-Gayon M.-D., 2011. La légitimité de la forêt des Landes de Gascogne du XIX ${ }^{\mathrm{e}}$ siècle à la tempête de 2009, in Société de Borda (Ed.), Tempêtes sur la forêt landaise. Histoires, mémoires, Mont-de-Marsan, Atelier des Brisants, 165-181.

Robinet C., Van Opstal N., Baker R., Roques A., 2011. Applying a spread model to identify the entry points from which the pine wood nematode would spread most rapidly across Europe, Biological Invasions, 13, 2981-2995, http:// dx.doi.org/10.1007/s10530-011-9983-0.

Roman-Amat B., 2007. Préparer les forêts françaises au changement climatique, Paris, ministère de l'Agriculture et de la Pêche, http://docs.gip-ecofor.org/libre/Roman-Amat foret-CC 2007.pdf.

Sewell W.J., 1996. Three temporalities: toward an eventful sociology, in McDonald T.J. (Ed.) The historic turn in the human sciences, Ann Arbor, University of Michigan Press, 245-280.

Citation de l'article : Banos V., Deuffic P. Après la catastrophe, bifurquer ou persévérer? Les forestiers à l'épreuve des événements climatiques extrêmes. Nat. Sci. Soc. 28, 3-4, 226-238. 